\title{
Genomic characterization of a pectinolytic isolate of Serratia oryzae isolated from lake water
}

\author{
Nicole Hugouvieux-Cotte-Pattat ${ }^{1}$, Cécile Jacot-des-Combes² ${ }^{2}$ Jérôme Briolay ${ }^{2}$ \\ 1. Univ Lyon, CNRS, INSA Lyon, Université Claude Bernard Lyon 1, UMR5240 Microbiologie Adaptation et Pathogénie, F-69621Villeurbanne, France \\ 2. Univ Lyon, Université Claude Bernard Lyon 1, CNRS, plateforme DTAMB, FR3728 BioEnviS, F-69621Villeurbanne, France \\ $\bowtie$ Corresponding author: E-mail: Nicole.Cotte-Pattat@insa-lyon.fr \\ (C) The author(s). This is an open access article distributed under the terms of the Creative Commons Attribution License (https://creativecommons.org/licenses/by/4.0/). \\ See http://ivyspring.com/terms for full terms and conditions.
}

Received: 2019.07.12; Accepted: 2019.09.16; Published: 2019.10.15

\begin{abstract}

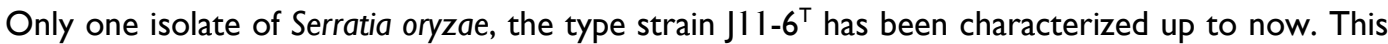
strain was found in the endophytic bacterial flora of rice. As part of an ongoing investigation into pectinolytic bacteria present in lake water in France, a few Serratia strains were isolated, including S32 and J9 identified as new strains of S. oryzae. The genome of strain S32 consists of a circular chromosome of 4,810,389 bp that contains 4,584 protein-coding genes. The genome of S32, as well as those of the type strain $\mathrm{J11}^{\mathrm{T}} \mathrm{6}^{\top}$, contains several genes involved in pectin degradation and in the intracellular assimilation of pectin oligomers. The specific detection of enzyme activities confirmed that strain S32 secretes functional pectinases and that it also produces extracellular cellulase and protease activities. The ability to produce plant cell wall degrading enzymes shows that $S$. oryzae shares characteristics of plant associated bacteria, including phytopathogens.
\end{abstract}

Key words: Serratia oryzae, isolate, lake water

\section{Introduction}

The Serratia genus is a member of the Yersiniaceae family, in the Enterobacterales order [1]. This family comprises seven genera Chania, Ewingella, Rahnella, Rouxiella, Samsonia, Serratia, and Yersinia [1]. Bacteria of the genus Serratia are common in the environment and were isolated from a variety of niches, including soil, water, air, plants, and animals [2]. Some species, such as $S$. rubidiae, S. ficaria and $S$. oryzae are closely associated with plants $[3,4,5]$. Other species, such as S. fonticola and S. aquatilis, occur naturally in aquatic environments $[6,7]$.

Our laboratory is interested in bacteria of the genus Dickeya, an important group of plant pathogens [8]. In the order Enterobacterales, Dickeya species belong to the family of Pectobacteriaceae that includes the five genera Brenneria, Dickeya, Lonsdalea, Pectobacterium, and Sodalis. Dickeya and Pectobacterium species provoke soft-rot diseases on a wide range of hosts, due to the action of extracellular pectinases that attack the plant cell wall [9]. They are frequently isolated from vegetable crops or ornamental plants, but also from water. To better understand the natural diversity of Dickeya species, we performed a survey of pectinolytic bacteria occurring in the water of small lakes of the French region La Dombes. Strain selection was based on a semi-selective solid medium which includes pectin as a gelling agent; this medium is commonly used for the isolation of Dickeya and Pectobacterium [10]. We found several pectinolytic isolates in lake water, including a new Dickeya species, D. lacustris [11]. However, preliminary genetic analysis indicated that some of the isolates did not belong to the Pectobacteriaceae family. For instance, the two isolates S32 and J9 showed a high pectinolytic activity and genetic analysis suggested that they belong to the Serratia genus and, most probably, to the species $S$. oryzae. Currently, only one S. oryzae strain, the type strain J11-6 ${ }^{\mathrm{T}}$, has been characterized [5]. This strain was found in the endophytic bacterial flora of rice. We sequenced the S32 genome to gain 
knowledge on the natural variability of $S$. oryzae and in the factors involved in its adaptation to the plant or aquatic environment.

\section{Methods}

\section{Isolation of pectinolytic bacteria}

Strains J9 and S32 were isolated in water from lakes of the wetland region La Dombes in France. The site of Foundation Pierre Vérots (latitude 45.951937 and longitude 4.883082) is reserved for the study and preservation of biodiversity. The anthropogenic contribution is minimal into this site. It contains four lakes surrounded by wet meadows and forests. Water samples were collected from the lakesides approximately $1.5 \mathrm{~m}$ from the shore and at less than $0.5 \mathrm{~m}$ depth. The water of the lakes is naturally eutrophic and rich in plant debris.

A first filtration on pleated paper filters was used to remove large particles from the eutrophic water. Then, the samples were used directly or concentrated $30 \mathrm{x}$ by centrifugation for $10 \mathrm{~min}$ at $8000 \mathrm{~g}$ (the pellet of a $15 \mathrm{ml}$ sample was suspended in $0.5 \mathrm{ml}$ of sterile water). Aliquots of $100 \mu \mathrm{l}$ of water, before and after concentration, were spread onto crystal violet pectate (CVP) plates. After incubating for $48 \mathrm{~h}$ at $30^{\circ} \mathrm{C}$, pectinolytic bacteria were detected by the formation of cavities around colonies, due to the degradation of polygalacturonic acid [10]. Strain J9 was isolated in June 2017 from a water sample of lake Riquet (5 ha) at a temperature of $29.2{ }^{\circ} \mathrm{C}$. Strain S32 was isolated in September 2017 from a concentrated water sample of lake Boufflers (28 ha) at a temperature of $18.6^{\circ} \mathrm{C}$.

\section{Genomic DNA extraction, genome sequencing and analysis}

The total bacterial genomic DNA of strain S32 was extracted using a NucleoSpin ${ }^{R}$ bacterial DNA purification kit (Macherey-Nagel). Quantification and quality control of the DNA was performed using a Nanodrop spectrophotometer, a Qubit4 fluorometer and agarose gel electrophoresis. Genomic DNA was sequenced using a MiSeq Illumina platform (Biofidal France). RAST was used for automatic annotation of the genome [12]. This annotation was used to search for genes potentially involved in pectin catabolism. Polysaccharide lyases (PLs) and glycoside hydrolases (GHs) were classified according to CAZY (http://www.cazy.org/). Blast comparisons were carried out using the NCBI website (https://blast. ncbi.nlm.nih.gov/Blast.cgi). The prediction of signal peptides was performed using SignalP 5.0 (http://www.cbs.dtu.dk/services/SignalP/).

PCR amplifications were performed on bacterial cell lysates using an Illustra ${ }^{\mathrm{TM}}$ PuReTaq $^{\mathrm{TM}}$ Ready-
To-Go ${ }^{\mathrm{TM}}$ kit (GE Healthcare). To perform a preliminary strain identification, PCR amplification was performed using the primers gapA-7-F and gapA-938-R [13] and the gapA amplicon sequences were determined by Sanger sequencing (Biofidal France). The strain taxonomic position was further clarified by calculation of average nucleotide identity (ANI) values (http://enve-omics.ce.gatech.edu/ani/) [14]. These analyses were performed using the genomes of a selection of 24 well-characterized Serratia strains representative of 13 species and including 11 type strains.

Phylogenetic trees were constructed using the neighbour-joining method [15], with calculation of the percentages of replicate trees in which the associated taxa clustered together in the bootstrap test (1000 replicates) [16]. For DNA, the evolutionary distances (number of base substitutions per site) were computed using the maximum composite likelihood method [17]. For proteins, the evolutionary distances were computed using the Poisson correction method [18]. Evolutionary analyses were conducted in MEGA7 [19].

\section{Phenotypic characterization}

To test their growth with different carbon sources, strains were inoculated onto M63 minimal medium plates supplemented with the appropriate carbon source $\left(2 \mathrm{~g} \mathrm{l}^{-1}\right)$. Growth was recorded after incubating for 24 and $48 \mathrm{~h}$ at $30^{\circ} \mathrm{C}$.

Production of extracellular enzymes was detected on specific media. Pectinase secretion was detected after growth for $24 \mathrm{~h}$ at $30^{\circ} \mathrm{C}$ on plates containing M63 medium supplemented with glycerol $\left(2 \mathrm{~g} \mathrm{l}^{-1}\right)$ and polygalacturonate $\left(4 \mathrm{~g} \mathrm{l}^{-1}\right)$. After flooding the plates with a saturated solution of copper acetate, clear zones appear around the colonies able to degrade polygalacturonate [20]. Cellulase secretion was detected after growth for $24 \mathrm{~h}$ at $30^{\circ} \mathrm{C}$ on plates containing M63 medium supplemented with glycerol $\left(2 \mathrm{~g} \mathrm{l}^{-1}\right)$ and carboxy-methyl-cellulose $\left(10 \mathrm{~g} \mathrm{l}^{-1}\right)$. After flooding with congo red $\left(5 \mathrm{~g} \mathrm{l}^{-1}\right)$ and washing with 1 $\mathrm{M} \mathrm{NaCl}$, clear zones appear around the colonies able to degrade cellulose [20]. Protease secretion was tested on LB agar plates containing skim milk powder $\left(6.25 \mathrm{~g} \mathrm{l}^{-1}\right)$, and clear zones around colonies were recorded after plate incubation for 24 and $48 \mathrm{~h}$ at $30^{\circ} \mathrm{C}$ [20]. Lecithinase secretion was tested on LB agar plates containing $5 \%$ egg yolk, and opalescent zones around colonies were recorded after plate incubation for 24 and $48 \mathrm{~h}$ at $30^{\circ} \mathrm{C}$ [21]. DNase secretion was tested on M63 agar plates containing salmon sperm DNA $\left(1 \mathrm{~g} \mathrm{l}^{-1}\right)$ and methyl green $\left(0.2 \mathrm{~g} \mathrm{l}^{-1}\right)$; light-green zones around colonies were recorded after plate incubation for $48 \mathrm{~h}$ at $30^{\circ} \mathrm{C}$ [22]. 
Table 1. ANI values between strain S32 and a selection of Serratia strains. This analysis was performed using the genomes of the strains S. ficaria WS3237 (NZ_QTUE01000001.1) and NCTC 12148' (LT906479.1), S. fonticola AU-AP2C (ASZA01000001.1) and LMG 7882 (AVAH01000001.1), S. grimesii BXF1 (LT883155.1) and NBRC 13537 (BCTT01000001.1), S. liquefaciens HUMV-21 (CP011303.1) and ATCC 27592 ${ }^{\top}$ (CP006252.1), S. marcescens B3R3 (CP011303.1) and NTCT 10211T (UGYK01000001.1), S. odorifera FDAARGOS 353 (NJFR02000001.1) and NCTC 11214' (NZ_LR134117.1), S. oryzae J11-6T (MOXD01000001.1), S. plymuthica NBRC 102599T (BCTU01000001.1) and AS9 (CP002773.1), S. proteamaculans B-41162 (MQMT01000001.1) and MPFA44A14 (FWWG01000001.1), S. quinivorans NCTC 11544T (UGYN01000001.1) and PKL12 (CP038467.1), S. rubidaea 1122 (CP014474.1) and NBRC 103169' (BCZJ01000001.1), S. symbiotica CWBI-2 (CCES01000001.1), S. ureilytica Lr5-4 (JSFB01000001.1) and DW2 (PGPC01000001.1).

\begin{tabular}{|c|c|c|c|c|c|c|c|c|c|c|c|c|c|c|c|c|c|c|c|c|c|c|c|c|c|}
\hline & 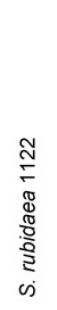 & 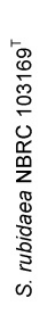 & 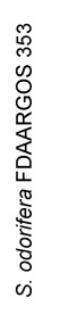 & 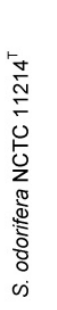 & 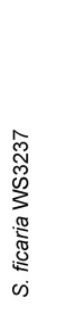 & 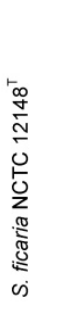 & 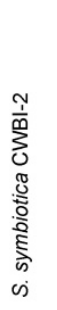 & 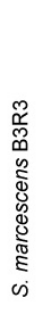 & 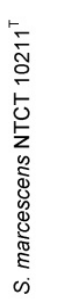 & 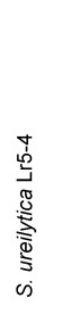 & 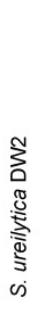 & 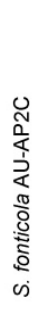 & 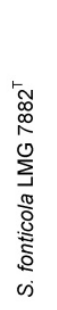 & 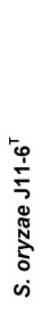 & 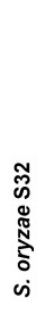 & 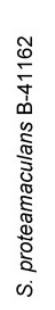 & 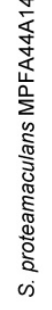 & 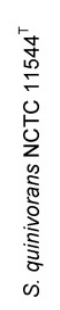 & 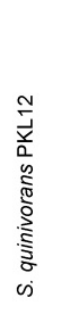 & 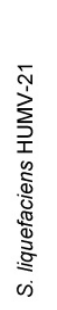 & 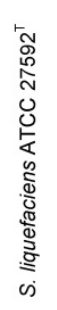 & 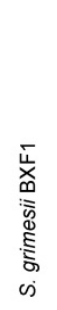 & 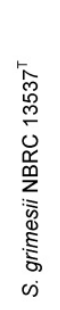 & 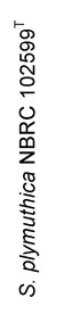 & 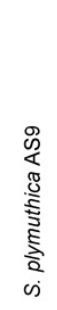 \\
\hline $\begin{array}{l}\text { S. rubidaea } \\
1122\end{array}$ & 100 & 99 & 83 & 83 & 84 & 84 & 81 & 84 & 84 & 84 & 84 & 81 & 82 & 82 & 82 & 82 & 82 & 82 & 82 & 82 & 82 & 81 & 81 & 83 & 83 \\
\hline $\begin{array}{l}\text { S. rubidaea } \\
\text { NBRC } 103169^{\top}\end{array}$ & 99 & 100 & 83 & 83 & 84 & 84 & 81 & 84 & 84 & 84 & 84 & 81 & 82 & 82 & 82 & 82 & 82 & 82 & 82 & 82 & 82 & 81 & 81 & 83 & 83 \\
\hline $\begin{array}{l}\text { S. odorifera } \\
\text { FDAARGOS } 353\end{array}$ & 83 & 83 & 100 & 100 & 83 & 83 & 82 & 83 & 83 & 83 & 83 & 81 & 82 & 81 & 81 & 82 & 82 & 82 & 82 & 82 & 82 & 81 & 81 & 82 & 82 \\
\hline $\begin{array}{l}\text { S. odorifera } \\
\text { NCTC } 11214^{\top}\end{array}$ & 83 & 83 & 100 & 100 & 83 & 83 & 81 & 83 & 83 & 83 & 83 & 81 & 82 & 81 & 81 & 82 & 82 & 82 & 82 & 82 & 82 & 81 & 81 & 82 & 82 \\
\hline $\begin{array}{l}\text { S. ficaria } \\
\text { WS3237 }\end{array}$ & 84 & 84 & 83 & 83 & 100 & 100 & 85 & 88 & 88 & 88 & 87 & 82 & 83 & 83 & 83 & 85 & 85 & 85 & 85 & 85 & 85 & 83 & 83 & 86 & 86 \\
\hline $\begin{array}{l}\text { S. ficaria } \\
\text { NCTC } 12148^{\top}\end{array}$ & 84 & 84 & 83 & 83 & 100 & 100 & 84 & 88 & 88 & 88 & 87 & 82 & 83 & 83 & 83 & 85 & 85 & 85 & 85 & 85 & 85 & 83 & 83 & 86 & 86 \\
\hline $\begin{array}{l}\text { S. symbiotica } \\
\text { CWBI-2 }\end{array}$ & 81 & 81 & 82 & 81 & 85 & 84 & 100 & 83 & 83 & 83 & 83 & 81 & 81 & 81 & 81 & 82 & 82 & 82 & 82 & 82 & 82 & 82 & 82 & 83 & 83 \\
\hline $\begin{array}{l}\text { S. marcescens } \\
\text { B3R3 }\end{array}$ & 84 & 84 & 83 & 83 & 88 & 88 & 83 & 100 & 97 & 95 & 95 & 82 & 82 & 82 & 82 & 83 & 84 & 84 & 84 & 84 & 84 & 82 & 82 & 84 & 84 \\
\hline $\begin{array}{l}\text { S. marcescens } \\
\text { NTCT } 10211^{\top}\end{array}$ & 84 & 84 & 83 & 83 & 88 & 88 & 83 & 97 & 100 & 95 & 95 & 82 & 82 & 82 & 82 & 83 & 84 & 84 & 84 & 84 & 84 & 82 & 82 & 84 & 84 \\
\hline $\begin{array}{l}\text { S. ureilytica } \\
\text { Lr5-4 }\end{array}$ & 84 & 84 & 83 & 83 & 88 & 88 & 83 & 95 & 95 & 100 & 100 & 82 & 82 & 82 & 82 & 83 & 84 & 84 & 84 & 84 & 84 & 82 & 82 & 84 & 84 \\
\hline $\begin{array}{l}\text { S. ureilytica } \\
\text { DW2 }\end{array}$ & 84 & 84 & 83 & 83 & 87 & 87 & 83 & 95 & 95 & 100 & 100 & 82 & 82 & 82 & 82 & 83 & 84 & 84 & 84 & 84 & 84 & 82 & 82 & 84 & 84 \\
\hline $\begin{array}{l}\text { S. fonticola } \\
\text { AU-AP2C }\end{array}$ & 81 & 81 & 81 & 81 & 82 & 82 & 81 & 82 & 82 & 82 & 82 & 100 & 96 & 83 & 83 & 82 & 82 & 82 & 82 & 82 & 82 & 81 & 81 & 82 & 82 \\
\hline $\begin{array}{l}\text { S. fonticola } \\
\text { LMG } 7882^{\top}\end{array}$ & 82 & 82 & 82 & 82 & 83 & 83 & 81 & 82 & 82 & 82 & 82 & 96 & 100 & 83 & 83 & 82 & 82 & 82 & 82 & 82 & 82 & 82 & 82 & 82 & 82 \\
\hline $\begin{array}{l}\text { S. oryzae } \\
\text { J11-6 }\end{array}$ & 82 & 82 & 81 & 81 & 83 & 83 & 81 & 82 & 82 & 82 & 82 & 83 & 83 & 100 & 99 & 82 & 82 & 82 & 82 & 82 & 82 & 82 & 82 & 82 & 82 \\
\hline $\begin{array}{l}\text { S. oryzae } \\
\text { S32 }\end{array}$ & 82 & 82 & 81 & 81 & 83 & 83 & 81 & 82 & 82 & 82 & 82 & 83 & 83 & 99 & 100 & 82 & 82 & 82 & 82 & 82 & 82 & 81 & 82 & 82 & 82 \\
\hline $\begin{array}{l}\text { S. proteamaculans } \\
\text { B-41162 }\end{array}$ & 82 & 82 & 82 & 82 & 85 & 85 & 82 & 83 & 83 & 83 & 83 & 82 & 82 & 82 & 82 & 100 & 96 & 91 & 91 & 87 & 87 & 87 & 87 & 86 & 86 \\
\hline $\begin{array}{l}\text { S. proteamaculans } \\
\text { MPFA44A14 }\end{array}$ & 82 & 82 & 82 & 82 & 85 & 85 & 82 & 84 & 84 & 84 & 84 & 82 & 82 & 82 & 82 & 96 & 100 & 91 & 91 & 87 & 87 & 87 & 87 & 87 & 87 \\
\hline $\begin{array}{l}\text { S. quinivorans } \\
{\text { NCTC } 11544^{\top}}\end{array}$ & 82 & 82 & 82 & 82 & 85 & 85 & 82 & 84 & 84 & 84 & 84 & 82 & 82 & 82 & 82 & 91 & 91 & 100 & 99 & 87 & 87 & 87 & 87 & 86 & 86 \\
\hline $\begin{array}{l}\text { S. quinivorans } \\
\text { PKL12 }\end{array}$ & 82 & 82 & 82 & 82 & 85 & 85 & 82 & 84 & 84 & 84 & 84 & 82 & 82 & 82 & 82 & 91 & 91 & 99 & 100 & 87 & 87 & 87 & 87 & 86 & 86 \\
\hline $\begin{array}{l}\text { S. liquefaciens } \\
\text { HUMV-21 }\end{array}$ & 82 & 82 & 82 & 82 & 85 & 85 & 82 & 84 & 84 & 84 & 84 & 82 & 82 & 82 & 82 & 87 & 87 & 87 & 87 & 100 & 99 & 86 & 86 & 86 & 86 \\
\hline $\begin{array}{l}\text { S. liquefaciens } \\
{\text { ATCC } 27592^{\top}}\end{array}$ & 82 & 82 & 82 & 82 & 85 & 85 & 82 & 84 & 84 & 84 & 84 & 82 & 82 & 82 & 82 & 87 & 87 & 87 & 87 & 99 & 100 & 85 & 86 & 86 & 86 \\
\hline $\begin{array}{l}\text { S. grimesii } \\
\text { BXF1 }\end{array}$ & 81 & 81 & 81 & 81 & 83 & 83 & 82 & 82 & 82 & 82 & 82 & 81 & 82 & 82 & 81 & 87 & 87 & 87 & 87 & 86 & 85 & 100 & 99 & 85 & 85 \\
\hline $\begin{array}{l}\text { S. grimesii } \\
\text { NBRC } 13537^{\top}\end{array}$ & 81 & 81 & 81 & 81 & 83 & 83 & 82 & 82 & 82 & 82 & 82 & 81 & 82 & 82 & 82 & 87 & 87 & 87 & 87 & 86 & 86 & 99 & 100 & 85 & 85 \\
\hline 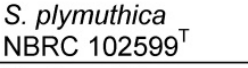 & 83 & 83 & 82 & 82 & 86 & 86 & 83 & 84 & 84 & 84 & 84 & 82 & 82 & 82 & 82 & 86 & 87 & 86 & 86 & 86 & 86 & 85 & 85 & 100 & 97 \\
\hline $\begin{array}{l}\text { S. plymuthica } \\
\text { AS9 }\end{array}$ & 83 & 83 & 82 & 82 & 86 & 86 & 83 & 84 & 84 & 84 & 84 & 82 & 82 & 82 & 82 & 86 & 87 & 86 & 86 & 86 & 86 & 85 & 85 & 97 & 100 \\
\hline
\end{tabular}




\section{Bacterial motility and maceration ability}

The swimming motility, inside the soft agar medium, was estimated by pricking 3 bacterial colonies with a thin rod inside a $0.3 \%$ GL agar plate which was then incubated at $30^{\circ} \mathrm{C}$ for $24 \mathrm{~h}$ before measuring the growth diameter.

The swarming motility, at the surface of the agar medium, was estimated by inoculating 3 drops of $2 \mu \mathrm{l}$ of a fresh bacterial culture on a $0.6 \%$ GL agar plate supplemented with glucose $\left(5 \mathrm{~g} \mathrm{l}^{-1}\right)$. The plates were incubated at $30^{\circ} \mathrm{C}$ for $24 \mathrm{~h}$ before measuring colony expansion. Surfactant production was visualized by the presence of a transparent film on the plate surface around the growth zone.

To test the maceration capacity on plant tissues, we inoculated chicory leaves and potato tubers [23]. Seven chicory leaves were infected for each strain, using $10 \mu \mathrm{l}$ of bacterial suspension $\left(10^{9} \mathrm{cfu} / \mathrm{ml}\right.$ in $\mathrm{M} 63$ medium). After incubation in a humid chamber for 24 $\mathrm{h}$ at $30^{\circ} \mathrm{C}$, the length of macerated tissue was measured. Ten potato tubers were inoculated with 5 $\mu \mathrm{l}$ of bacterial suspension $\left(10^{9} \mathrm{cfu} / \mathrm{ml}\right.$ in $\mathrm{M} 63$ medium). Following incubation for $48 \mathrm{~h}$ at $30^{\circ} \mathrm{C}$ in a humid chamber, the weight of rotten tissue was measured to estimate the maceration ability. The model Dickeya strain, D. dadantii 3937, was used as a positive control in these experiments. Negative controls were performed using M63 medium.

\section{Results and Discussion}

The high pectinolytic activity of the two isolates S32 and J9 justified their careful identification. As a first approach, their gapA gene was amplified by PCR and sequenced. Analysis of the gap A amplicons showed a close proximity between strains J9 and S32 (99\% identity). Blast search against $\mathrm{nr} / \mathrm{nt} \mathrm{NCBI}$ databank indicated that the best scores (94 to 95\% identities) for the J9 or S32 gapA amplicons are found with strains of the genus Serratia, including several strains of $S$. fonticola, S. liquefaciens, S. proteamaculans, S. quinivorans, and of non-identified Serratia species. The level of identity with strains of other characterized Serratia species is lower than $90 \%$. To gain information on this water isolate, the genome sequence of strain S32 was determined. The draft genome comprises 149 contigs ( $\mathrm{N} 50=119,595, \mathrm{~L} 50=14$, $324 X$ coverage depth) with a total length of $4,810,389$ bp and a $\mathrm{G}+\mathrm{C}$ content (mol\%) of $53.3 \%$. The S32 genome was automatically annotated with RAST [12], predicting 4,584 protein coding genes and 84 RNA-coding sequences, including 75 tRNAs and 9 rRNAs $(23 S, 16 S$, and $7 \times 5 S)$.

The most relevant parameter for ascertaining the identity of a strain is the calculation of ANI that measure the genetic distance between two genomes [14]. The taxonomic identification of strain S32 was clarified by calculation of ANI values based on pairwise comparisons between the S32 genome and a selection of 24 genomes of characterized Serratia species. An ANI value higher than 99\% was found with S. oryzae J11-6 $6^{\mathrm{T}}$ and ANI values of $81-83 \%$ were observed with other Serratia species (Table 1). According to a threshold of $95 \%$ for delineating the species boundaries [14], S32 belongs to the species $S$. oryzae. A new Blast search against the J11- $6^{\mathrm{T}}$ genome (NCBI assembly GCA_001976145.1) using the sequence of the S32 or J9 gapA amplicons showed that each gap $A$ sequence shares more than $99 \%$ identity with J11-6 ${ }^{\mathrm{T}}$ DNA, confirming the close proximity between the three strains (Figure 1).

A high level of homology was found between the genome of S32 and that of the type strain of S. oryzae, J11-6 ${ }^{\mathrm{T}}$. The complete genome sequence of $S$. oryzae J11- $6^{\mathrm{T}}$ consists of 5,012,778 bp, with $\mathrm{G}+\mathrm{C}$ contents of $53.2 \%$ and 4,733 predicted protein coding genes by automatic annotation with RAST. Using the function-based comparison tool in RAST, 88 genes were predicted to be present in S32 but absent in J11- $6^{\mathrm{T}}$ and, inversely, 64 genes were predicted in $\mathrm{J} 11-6^{\mathrm{T}}$ but absent in S32. Several S32 specific genes are related to phages or mobile elements (25 genes), or predicted to encode fimbriae (5 genes), toxins/ antitoxins (5 genes), or restriction-modification systems (5 genes) (Table S1). Several genes present in J11- $6^{\mathrm{T}}$ but absent in S32 also correspond to phages or mobile elements (21 genes); others are predicted to encode proteins involved in adhesion (11 genes), urease subunits (8 genes), or CRISPR- associated proteins (7 genes) (Table S2). Such strains specific genes often occur in clusters, suggesting horizontal acquisition.

Based on the RAST annotations, we searched for genes potentially involved in pectin catabolism. Similar genes were found in both S32 and J11-6 genomes, reflecting their ability in degrading this plant polysaccharide. Both strains contain genes encoding a predicted pectate lyase of the family PL1, three putative polygalacturonases of the family GH28 and an oligogalacturonate lyase of the family PL22. Among the sequenced Serratia strains, a few other genomes contain potential pectinase genes (http://www.cazy.org/). Some S. fonticola and S. odorifera genomes are predicted to encode a PL9 pectate lyase, a PL22 oligogalacturonate lyase and one or two GH28 polygalacturonases. However, among the sequenced Serratia strains, proteins of the family PL1 are rare and found only in S. oryzae J11-6 ${ }^{\mathrm{T}}$ and a few strains of non-characterized species (Figure 2). In contrast, in living organisms, PL1 is the largest family 
of pectate lyases, including several hundred representatives from different kingdoms, plants, fungi, oomycetes, bacteria and archaea [24]. Plant-pathogenic bacteria often possess several PL1 members which have a high specific activity. For instance, the phytopathogen D. dadantii 3937 encodes eight PL1 proteins, including six characterized pectate lyases and two putative pectin lyases [24]. The PL1 pectate-lyase of S32, Pel1, shares homology with proteins of $S$. oryzae J11-6 $(99.8 \%$ identity), five non-identified Serratia strains (Ag1, Ag2, H1n, H1w, and 3ACOL1,with 91, 91, 78, 78 and 76\% identity, respectively), Chania multitudinisentens (79\% identity) and diverse strains of three Yersinia species, $Y$. massiliensis, Y. frederiksenii and Y. intermedia (about $75 \%$ identity) (Figure 2). Outside these proteins from Enterobacterales, several Pel1 homologs are found in Gram positive bacteria such as Cellulosimicrobium and Cellulomonas (about 53 and $51 \%$ identity, respectively). None of these bacteria encoding a Pel1 homolog has been previously reported to be a phytopathogen. Comparison with proteins encoded by $D$. dadantii 3937 showed that the S. oryzae Pel1 shares about $30 \%$ identity with the major pectate lyases PelA, PelB, PelC, PelD and PelE of this plant pathogenic species [24].

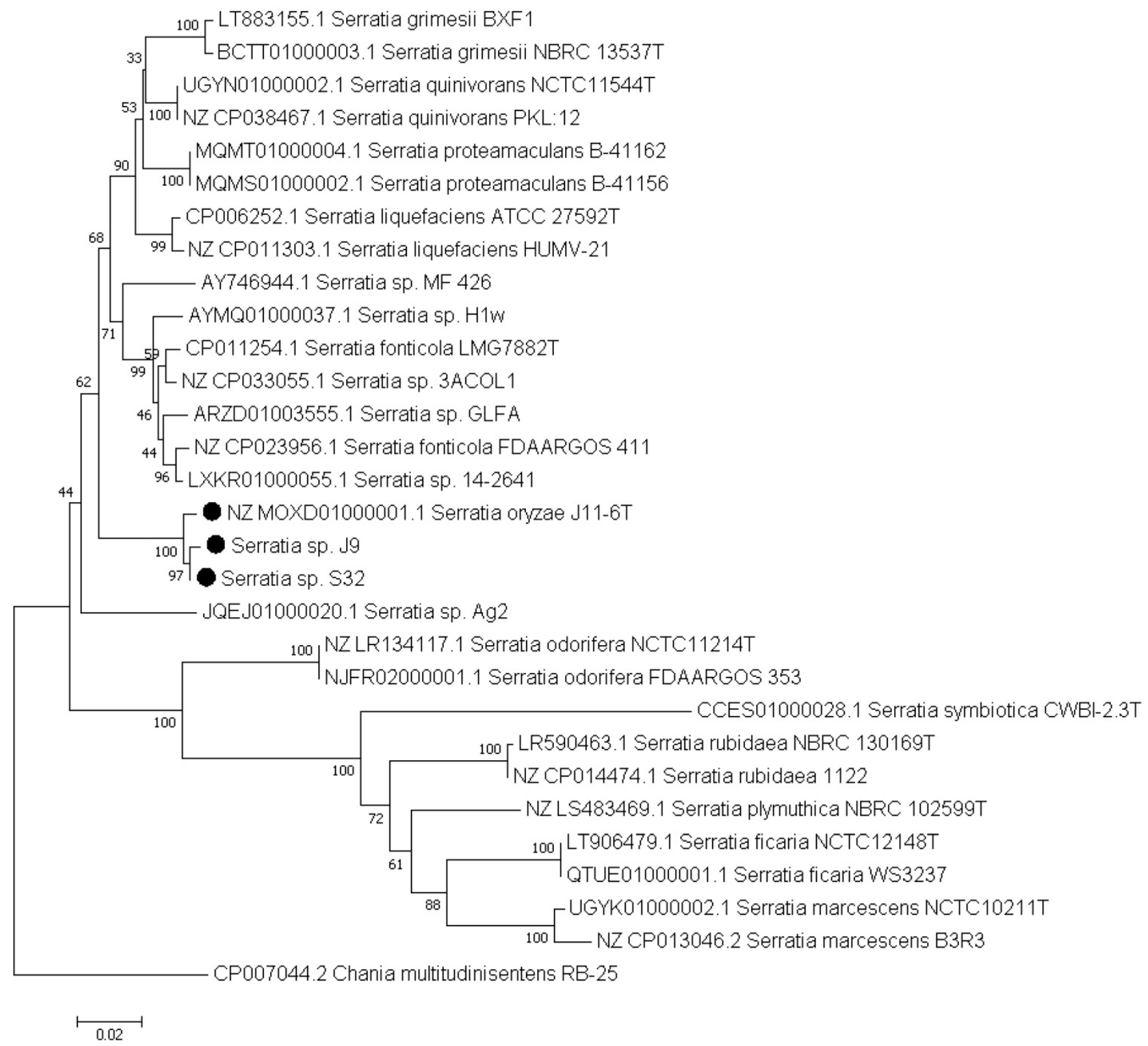

Figure 1. Phylogenetic position of strain S32 and $\mathbf{j 9}$ based on the gapA sequences. This analysis was performed using the gapA sequences of strains belonging to well-characterized Serratia species, and of Serratia sp. strains whose genome encodes a PLl pectate lyase. The gene gapA of Chania multitudinisentens was used as an outgroup. The evolutionary history was inferred using the neighbour-joining method [15]. The percentages of replicate trees in which the associated taxa clustered together in the bootstrap test are shown next to the branches [16]. The evolutionary distances were computed using the maximum composite likelihood method [17] and are in the units of the number of base substitutions per site (718 positions). Evolutionary analyses were conducted in MEGA7 [19]. 


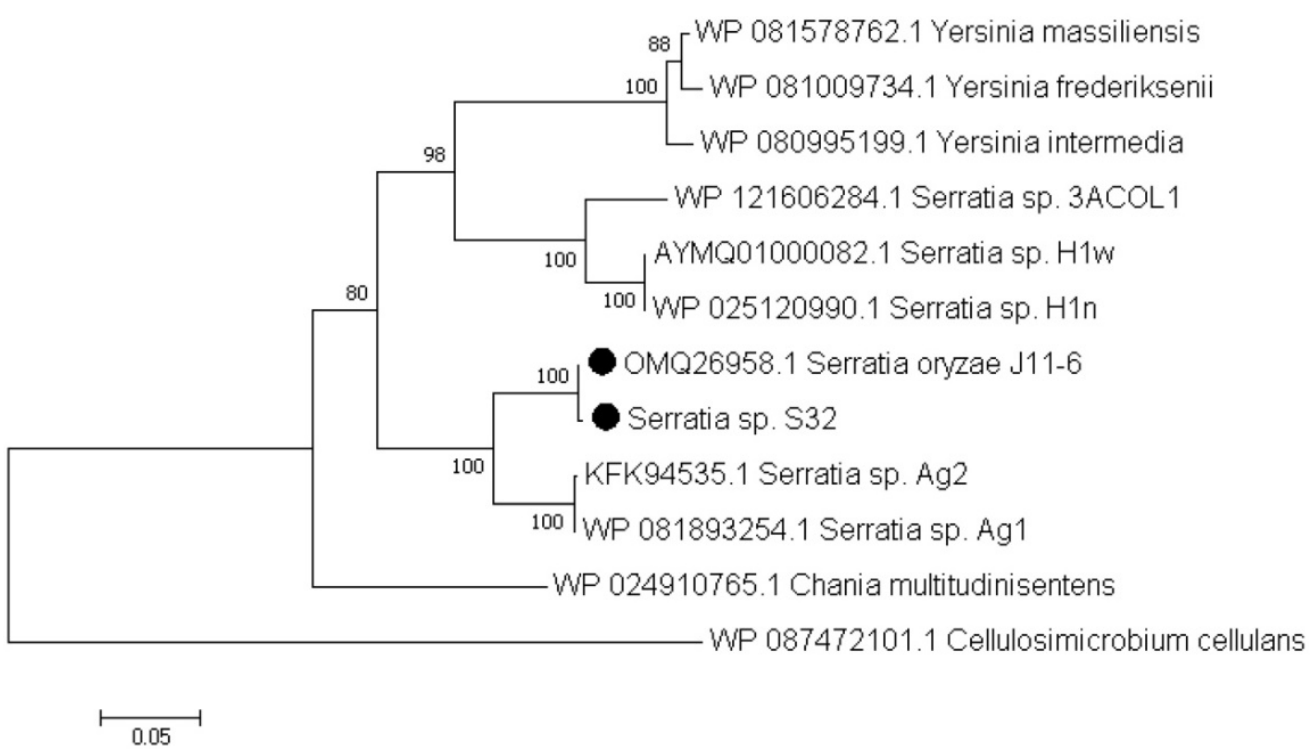

Figure 2. Phylogenetic tree of the PL1 protein Pell of strain S32. The evolutionary history was inferred using the neighbour-joining method [15]. The percentages of replicate trees in which the associated taxa clustered together in the bootstrap test (1000 replicates) are shown next to the branches [16]. The evolutionary distances were computed using the Poisson correction method [18] and are in the units of the number of amino acid substitutions per site (430 positions). Evolutionary analyses were conducted in MEGA7 [19]. This analysis was performed using the sequences of the different Serratia strains encoding a PL1 protein. A homologous protein of Cellulosimicrobium cellulans was used as an outgroup.

The pectate-lyase Pel1 of S32 presents a N-terminal signal peptide typical of precursor proteins transported by the $\mathrm{Sec}$ translocon and cleaved by Signal Peptidase I [25], suggesting its exportation in the bacterial periplasm. Enzymatic assays demonstrated the presence in the S32 culture supernatant of a pectate lyase activity able to cleave polygalacturonate, the pectin backbone. A gene cluster encoding a complete type II secretion system (T2SS) is present in S. oryzae genome; it could be involved in Pel1 secretion. T2SS mediates secretion of proteins from the periplasm to the external medium and a well-studied T2SS is responsible for specific pectate lyase secretion in the genus Dickeya [26]. Two GH 28 polygalacturonases of S32 present the $\mathrm{N}$-terminal lipoprotein signal peptide typical of proteins transported by the Sec translocon and cleaved by Signal Peptidase II [25]. Thus, these two proteins are predicted to be anchored to the bacterial outer membrane. Some $D$. dadantii pectinases have already been shown to be associated with the outer membrane, anchored either on the periplasmic or extracellular sides of this membrane $[27,28]$.

Both S32 and J11-6 ${ }^{\mathrm{T}}$ genomes have also all the genes necessary for intracellular pectin metabolism, encoding the transporters $\mathrm{KdgM}$, TogMNAB and $\operatorname{Tog} \mathrm{T}$ [29], and the enzymes KdgF, KduI, KduD, $\mathrm{KdgK}$, and $\mathrm{KdgA}[30,31]$ (Figure 3). All these catabolic steps are also encoded by the Dickeya genomes, allowing bacteria to efficiently use oligosaccharides resulting from degradation of the pectin backbone, as a sole carbon and energy source for growth [32].

Diverse compounds were independently added to minimal medium to test their utilization as a sole carbon source by strains S32 and J9. This phenotypic analysis confirms that the two strains are able to grow in the presence of polygalacturonate as a sole carbon source. They are also able to grow in the presence of several monosaccharides or disaccharides, such as D-arabinose, D-fructose, D-galactose, D-galacturonate, D-glucose, D-gluconate, D-glucuronate, m-inositol, D-mannitol, D-mannose, melibiose, L-rhamnose, D-ribose, sucrose, trehalose, and $\mathrm{D}$-xylose. The use of different biochemical tests for the specific detection of extracellular enzyme activities showed that both S32 and J9 secrete pectinase, cellulase and protease activities (Table 2). However, the two strains are not strictly identical; the enzymatic activities of J9 are higher than that of S32, particularly for cellulase and lecithinase production (Table 2). Since they produce extracellular pectate lyase activity, we tested the potential ability of these strains to macerate plant tissues (Table 3). S32 showed a significant ability to macerate plant tissues; the amount of rotten tissue obtained either on chicory leaves or potato tubers, was about one-third that obtained with the plant pathogen, $D$. dadantii (Table 3). Strain $S 32$ exhibited high swimming and swarming motilities and surfactant production (Table 3). Strain J9 showed a lower maceration capacity than S32, probably related to its lower motility (Table 3 ). 
Table 2. Production of extracellular enzymes by Serratia oryzae. D. dadantii 3937 was used as a reference strain. (+, positive; -, negative; w, weak)

\begin{tabular}{llll}
\hline & Serratia oryzae & & Dickeya dadantii \\
\hline & S32 & J9 & 3937 \\
Pectinase & + & + & + \\
Cellulase & $\mathrm{w}$ & + & + \\
Protease & + & + & + \\
Lecithinase & - & $\mathrm{w}$ & + \\
DNase & - & - & + \\
\hline
\end{tabular}

Table 3. Maceration ability and motility of Serratia oryzae. To estimate the maceration ability, the length of macerated tissue was measured $24 \mathrm{~h}$ after inoculation for chicory leaves and the weight of macerated tissue was measured after $48 \mathrm{~h}$ for potato tubers. The mean values are given with the standard deviations. To estimate the bacterial motility, the growth diameter was measured $24 \mathrm{~h}$ after inoculation in $0.3 \% \mathrm{GL}$ agar plate or on $0.6 \% \mathrm{GL}$ agar plate, respectively. Surfactant production was visualized by a transparent film on the plate surface. $D$. dadantii 3937 was used as a reference strain.

\begin{tabular}{llll}
\hline & \multicolumn{2}{l}{ Serratia oryzae } & Dickeya dadantii \\
\hline & $\mathrm{S} 32$ & $\mathrm{~J} 9$ & 3937 \\
Chicory leaf maceration $(\mathrm{mm})$ & $33 \pm 9$ & $10 \pm 4$ & $98 \pm 11$ \\
Potato tuber maceration $(\mathrm{g})$ & $1.26 \pm 0.45$ & $0.02 \pm 0.01$ & $3.69 \pm 0.68$ \\
Swimming motility $(\mathrm{mm})$ & $45 \pm 2$ & $5 \pm 1$ & $29 \pm 1$ \\
Swarming motility $(\mathrm{mm})$ & $35 \pm 4$ & $7 \pm 1$ & $26 \pm 4$ \\
Surfactant production & + & - & + \\
\hline
\end{tabular}

The previously characterized $S$. oryzae type strain, J11-6 ${ }^{\mathrm{T}}$, was found in the endophytic bacterial flora of rice [5]. The two new S. oryzae strains S32 and J9 were isolated from water of eutrophic lakes. They showed a high pectinolytic activity and production of plant cell wall degrading enzymes, indicating that $S$. oryzae strains share characteristics of plant associated bacteria, including phytopathogens. Since the two strains S32 and J9 are similar but not identical, it would be interesting to test the phenotypic features of the type strain $J 11-6^{\mathrm{T}}$ to gain insights in the natural diversity of $S$. oryzae strains. The $S$. oryzae genomes encode an extracellular pectate lyase of the PL1 family. These enzymes play various physiological roles; they are involved in the maceration of plant tissues caused by pectinolytic phytopathogens, in the establishment of symbiosis by rhizobia, in the degradation of plant litter in the soil, or in the digestion of vegetable food by gut bacteria. The $S$. oryzae pectate lyase could allow these bacteria to use pectin as a carbon source for growth and to efficiently degrade plant debris found in their close environment, soil or water.

A

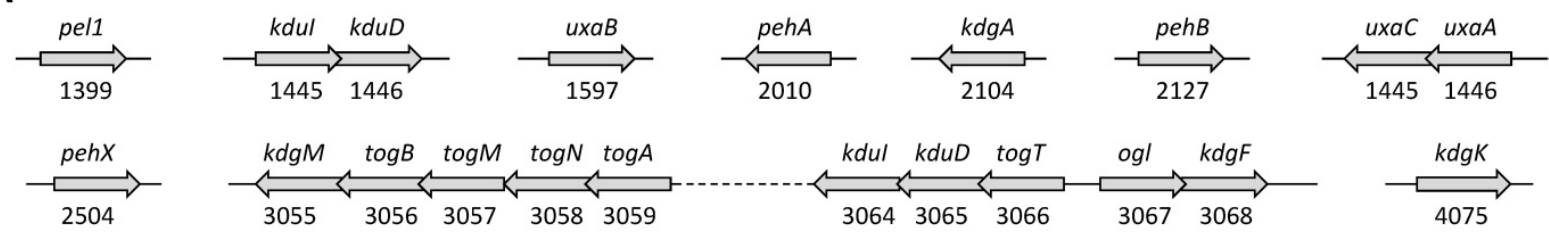

B

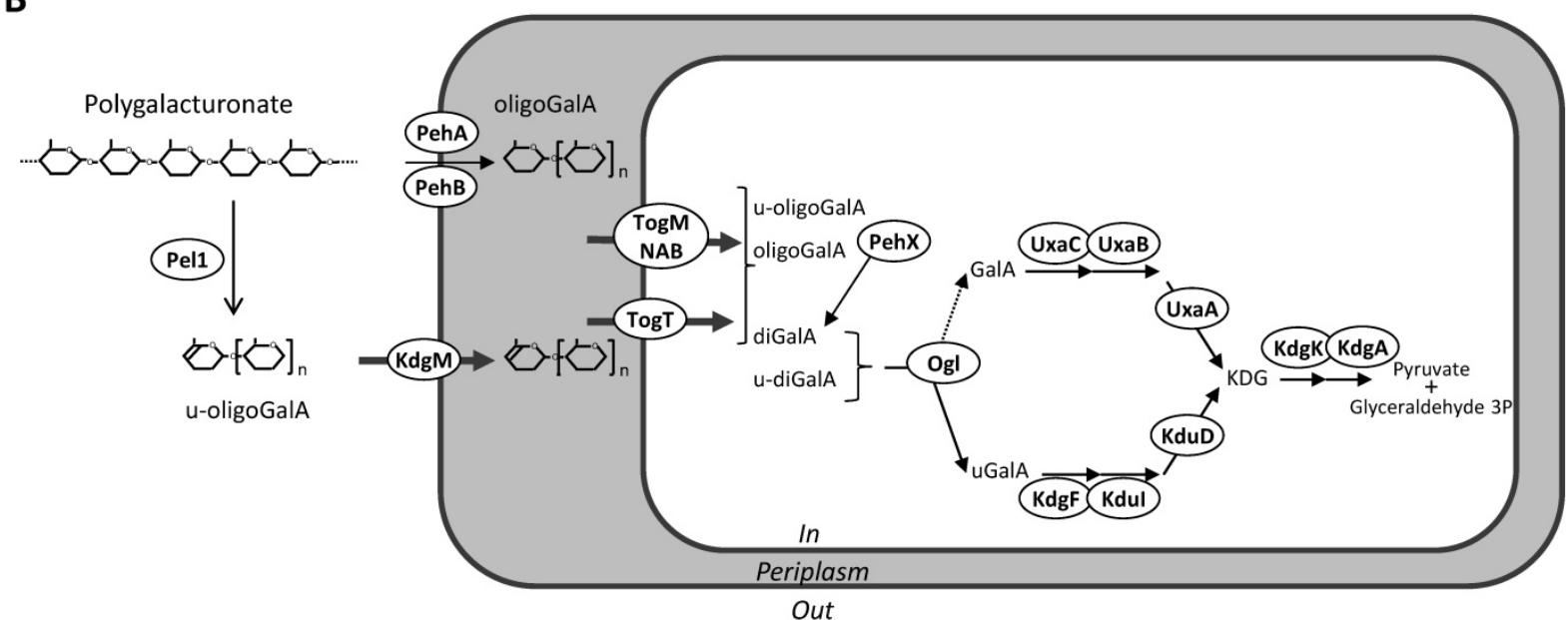

Figure 3. Genes and proteins of strain $\mathbf{S 3 2}$ potentially involved in pectin catabolism. A: Genetic organization. Genes are symbolized by arrows; their name and ID number are given. B: Proposed metabolic pathway deduced from the predicted protein localization and from the function of homologous proteins. The extracellularly pectate lyase Pell generates unsaturated oligogalacturonides (u-oligoGalA) up to dimers (u-diGalA, unsaturated digalacturonide). The activity of outer membrane anchored polygalacturonases, PehA and PehB, generates saturated oligomers up to dimers (oligoGalA, oligogalacturonides; diGalA, digalacturonide). Extracellular oligomers enter the periplasm using the specific outer-membrane porin, KdgM. Short oligomers enter the cytoplasm using two specific transporters, TogMNAB and TogT. In the cytoplasm, they are further cleaved up monomers by the action of the exopolygalacturonase PehX and the oligogalacturonate lyase, Ogl. The two pathways involved in the catabolism of galacturonate (GalA) and unsaturated galacturonate (uGalA) converge to produce a common intermediate, KDG (2-keto-3-deoxygluconate) which is converted to pyruvate and glyceraldehyde 3-phosphate. 


\section{Nucleotide sequence accession numbers}

This Whole Genome Shotgun project has been deposited at DDBJ/ENA/GenBank under the accession SOZF00000000. The S32 genome draft version described in this paper is version SOZF01000000.

\section{Abbreviations}

ANI: average nucleotide identity; CAZY: Carbohydrate-Active enZYmes; CVP: crystal violet pectate medium; GH: glycoside hydrolase; LB: Luria-Bertani medium; PSL: polysaccharide lyase; RAST: rapid annotations using subsystems technology; T2SS: type II secretion system.

\section{Supplementary Material}

Supplementary figures and tables.

http://www.jgenomics.com/v07p0064s1.pdf

\section{Acknowledgments}

We thank the staff of the Pierre Vérots Foundation for their warm welcome, and Véronique Utzinger for medium preparation and for assistance in implementation of phenotypic tests. This work was supported by the Pierre Vérots Foundation (http://www.fondation-pierre-verots.com/), FR3728 BioEnviS (AAP2017), Agence Nationale de la Recherche (ANR-15-CE21-0003), CNRS, University Lyon 1, and INSA Lyon. It has been benefited from the expertise and facilities of the platform DTAMB of FR3728 (Villeurbanne, France), with sequencing performed by BIOFIDAL (Vaux-en-Velin, France).

\section{Competing Interests}

The authors have declared that no competing interest exists.

\section{References}

1. Adeolu M, Alnajar S, Naushad S, Gupta RS. Genome-based phylogeny and taxonomy of the 'Enterobacteriales': proposal for Enterobacterales ord. nov. divided into the families Enterobacteriaceae, Erwiniaceae fam. nov., Pectobacteriaceae fam. nov., Yersiniaceae fam. nov., Hafniaceae fam. nov., Morganellaceae fam. nov., and Budviciaceae fam. nov. Int J Syst Evol Microbiol. 2016; 66: 5575-5599.

2. Petersen LM, Tisa LS. Friend or foe? A review of the mechanisms that drive Serratia towards diverse lifestyles. Can J Microbiol. 2013; 59: 627-640.

3. Grimont PAD, Grimont F, Starr MP. Serratia ficaria sp. nov., a bacterial species associated with smyrna figs and the fig wasp Blastophaga psenes. Curr Microbiol. 1979; 2: 277-282.

4. Grimont PAD, Grimont F, Starr MP. Serratia species isolated from plants. Curr Microbiol. 1981; 5: 317-322.

5. Zhang, C.W., J. Zhang, J.J. Zhao, X. Zhao, D.F. Zhao, H.Q. Yin and X.X. Zhang, Serratia oryzae sp. nov., isolated from rice stems. Int J Syst Evol Microbiol. 2017; 67: 2928-2933.

6. Kämpfer P, Glaeser SP. Serratia glossinae Geiger et al. 2010 is a later synonym of Serratia fonticola Gavini et al. 1979. Int J Syst Evol Microbiol. 2015; 65: 1406-1408.

7. Kämpfer P, Glaeser SP. Serratia aquatilis sp. nov., isolated from drinking water systems. Int J Syst Evol Microbiol. 2016; 66: 407-413.
8. Mansfield J, Genin S, Magori S, Citovky V, Sriariyanum M, Ronald P, Dow M, Verdier V, Beer SV, Machado MA, Toth I, Salmond G, Foster GD. Top 10 plant pathogenic bacteria in molecular plant pathology. Mol. Plant Pathol. 2012; 13:614-629.

9. Charkowski A, Blanco C, Condemine G, Expert D, Franza T, Hayes C, Hugouvieux-Cotte-Pattat N, Lopez Solanilla E, Low D, Moleleki L, Pirhonen M, Pitman A, Perna N, Reverchon S, Rodriguez-Palenzuela P, San Francisco M, Toth I, Tsuyumu S, van der Walls J, Van der Wolf J, van Gijsegem F, Yang CH, Yedidia I. The role of secretion systems and small molecules in soft rot Enterobacteriaceae pathogenicity. Annu Rev Phytopathol. 2012; 50: 425-449.

10. Hélias V, Hamon P, Huchet E, van der Wolf J, Andrivon D. Two new effective semiselective crystal violet pectate media for isolation of Pectobacterium and Dickeya. Plant Pathol. 2012; 61: 339-345.

11. Hugouvieux-Cotte-Pattat N, Jacot-des-Combes C, Briolay J. Dickeya lacustris sp. nov., a water-living pectinolytic bacterium isolated from lakes in France. Int J Syst Evol Microbiol. 2019; 69: 721-726.

12. Aziz RK, Bartels D, Best AA, DeJongh M, Disz T, Edwards RA, Formsma K, Gerdes S, Glass EM, Kubal M, Meyer F, Olsen GJ, Olson R, Osterman AL, Overbeek RA, McNeil LK, Paarmann D, Paczian T, Parrello B, Pusch GD, Reich C, Stevens R, Vassieva O, Vonstein V, Wilke A, Zagnitko O. The RAST server: rapid annotations using subsystems technology. BMC Genomics. 2008; 9: 75.

13. Cigna J, Dewaegeneire P, Beury A, Gobert V, Faure D. A gapA PCR sequencing assay for identifying the Dickeya and Pectobacterium potato pathogens. Plant Disease. 2017; 101: 1278-1282.

14. Goris J, Konstantinidis KT, Klappenbach JA, Coenye T, Vandamme P, Tiedje JM. DNA-DNA hybridization values and their relationship to whole genome sequence similarities. Int J Syst Evol Microbiol. 2007; 57: 81-91.

15. Saitou N, Nei M. The neighbour-joining method: A new method for reconstructing phylogenetic trees. Mol Biol Evol. 1987; 4: 406-425.

16. Felsenstein J. Confidence limits on phylogenies: An approach using the bootstrap. Evolution. 1985; 39: 783-791.

17. Tamura K, Nei M, Kumar S. Prospects for inferring very large phylogenies by using the neighbour-joining method. Proc Natl Acad Sci USA. 2004; 101: 11030-11035.

18. Zuckerkandl E, Pauling L. Evolutionary divergence and convergence in proteins. In: V Bryson and HJ Vogel eds. Evolving Genes and Proteins, pp. 97-166. Academic Press, New York. 1965.

19. Kumar S, Stecher G, Tamura K. MEGA7: Molecular Evolutionary Genetics Analysis version 7.0 for bigger datasets. Mol Biol Evol. 2016; 33: 1870-1874.

20. Ji J, Hugouvieux-Cotte-Pattat N, Robert-Baudouy J. Use of Mu-lac insertions to study the secretion of pectate lyases by Erwinia chrysanthemi. J Gen Microbiol. 1987; 133: 793-802.

21. Keen NT, Ridgway D, Boyd C. Cloning and characterization of a phospholipase gene from Erwinia chrysanthemi EC16. Mol Microbiol. 1992; 6: 179-187.

22. Moulard M, Condemine G, Robert-Baudouy J. Characterization of the $n u c M$ gene coding for a nuclease of the phytopathogenic bacteria Erwinia chrysanthemi. Mol Microbiol. 1993; 8: 685-695.

23. Hugouvieux-Cotte-Pattat N. The RhaS activator controls the Erwinia chrysanthemi 3937 genes rhiN, rhiT, and rhiE involved in rhamnogalacturonan catabolism. Mol Microbiol. 2004; 51: 1361-1374.

24. Hugouvieux - Cotte - Pattat N, Condemine G, Shevchik VE. Bacterial pectate lyases, structural and functional diversity. Environ Microbiol. Rep 2014; 6: 427-440.

25. Almagro Armenteros JJ, Tsirigos KD, Sønderby CK, Petersen TN, Winther O, Brunak S, von Heijne G, Nielsen H. SignalP 5.0 improves signal peptide predictions using deep neural networks. Nature Biotechnol. 2019; 37: 420-423.

26. Pineau C, Guschinskaya N, Robert X, Gouet P, Ballut L, Shevchik VE. Substrate recognition by the bacterial type II secretion system: more than a simple interaction. Mol Microbiol. 2014; 94: 126-140.

27. Shevchik VE, Condemine G, Hugouvieux-Cotte-Pattat N, Robert-Baudouy J. Characterization of pectin methylesterase B, an outer membrane lipoprotein of Erwinia chrysanthemi. Mol Microbiol. 1996; 19: 455-466.

28. Rondelet A., Condemine G. Type II secretion: The substrates that won't go away. Res Microbiol. 2013; 164: 556-561.

29. Hugouvieux-Cotte-Pattat N, Reverchon S. Two transporters, TogT and TogMNAB, are responsible for oligogalacturonide uptake in Erwinia chrysanthemi 3937. Mol Microbiol. 2001; 41: 1125-1132

30. Hugouvieux-Cotte-Pattat N, Condemine G, Nasser W, Reverchon S. Regulation of pectinolysis in Erwinia chrysanthemi. Annu Rev Microbiol. 1996; 50: 213-257.

31. Hobbs JK, Lee SM, Robb M, Hof F, Barr C, Abe KT, Hehemann JH, McLean R, Abbott DW, Boraston $\mathrm{AB}$. KdgF, the missing link in the 
microbial metabolism of uronate sugars from pectin and alginate. Proc Natl Acad Sci USA. 2016; 113: 6188-6193.

32. Hugouvieux-Cotte-Pattat N. Metabolism and virulence strategies in Dickeya-host interactions. Prog Mol Biol Transl Sci. 2016; 142: 93-129. 